\title{
CENTERS AND NEAREST POINTS OF SETS
}

\author{
P. SZEPTYCKI AND F. S. VAN VLECK
}

\begin{abstract}
For a Banach space $X$ and a subset $A$ of $X, c_{A}$ denotes the Čebyšev center of $A$ and $P_{A} x$ denotes the nearest point in $A$ to the point $x$ in $X$. The space of all subsets of $X$ is furnished with the Hausdorff metric. The modulus of continuity of the function $A \rightarrow c_{A}$ is computed in the case when $X$ is a Hilbert space and the sets $A$ are compact; the same is done for the function $A \rightarrow P_{A} x$, for fixed $x$, in the case when $X$ is uniformly convex and the sets $A$ are convex and closed.
\end{abstract}

1. Introduction. Let $X$ be a normed space and $A$ be a subset of $X$. Define $r_{A}=\inf \left\{r>0: \sup _{a \in A}\|x-a\| \leqslant r\right.$ for some $\left.x \in X\right\}$ - this is the radius of any circumsphere of $A$. If such a sphere exists we denote its center by $c_{A} ; c_{A}$ is called a Čebyšev center of $A$ (see [1]). If, in the supremum above, $x$ is restricted to a subspace of $X$, one obtains the notions of relative radius and of relative centers of $A$.

For any $x \in X$ we denote by $P_{A} x$ the set of all nearest points in $A$ to $x$, provided such points exist. $P_{A}$ is called the metric projection on $A$.

Without additional hypotheses on $X$ and $A, c_{A}, P_{A} x$ may not be defined and, even if they are, the maps $A \rightarrow c_{A}$ and $A \rightarrow P_{A} x$ may be multivalued.

There has been a number of recent papers on the subject of Čebyšev centers and metric projections; see for instance [7-15].

On the space $2^{X}$ of all subsets of $X$ we consider the (extended valued) Hausdorff metric

$$
h(A, B)=\inf \left\{\varepsilon>0: A \subset B^{\varepsilon} \text { and } B \subset A^{\varepsilon}\right\}
$$

where $A^{\varepsilon}$ denotes the $\varepsilon$-neighborhood of $A$.

The aim of this paper is to obtain an insight into the quantitative continuity properties of the functions $A \in 2^{X} \rightarrow c_{A} \in X$ and $A \in 2^{X} \rightarrow P_{A} x \in X$, for fixed $x$. The qualitative continuity properties have been studied in some of the references cited above.

The original motivation for this investigation was the hope that, with suitable hypotheses on $X$ and assuming convexity of the sets $A$, either or both of the above functions would provide a canonical selector for convex valued multifunctions which would inherit any continuity properties of the original multifunctions. Even though this expectation has not been fulfilled the partial results obtained seem to be of some interest in their own right.

Received by the editors January 3, 1980 and, in revised form, August 14, 1981.

AMS (MOS) subject classifications (1970). Primary 46B99, 46C 10.

Key words and phrases. Čebyšev center, nearest point, modulus of continuity.

(c) 1982 American Mathematical Society 0002-9939/81/0000-1110/\$02.25 
In the case when the closed unit ball in $X$ is compact in a suitable weak topology (e.g. when $X$ is a dual space), a center of any bounded set in $X$ exists. If $X$ is uniformly convex then such a center is unique [2]. In order that an arbitrary convex compact subset $A$ of $X$ have a center of its circumsphere contained in $A$ it is necessary and sufficient that $X$ be a Hilbert space $[3,4]$. In order that the (multi-) function $A \rightarrow c_{A}$ have a selector uniformly continuous on bounded sets of its domain it is necessary and sufficient that $X$ be uniformly convex [15].

A different notion of the center of a convex set (which coincides with the Čebyšev center in the case when $X$ is a Hilbert space) is given in [5].

The authors gratefully acknowledge the useful comments and contributions brought by the referee of this paper.

\section{Statement of results.}

THEOREM 1. If $X$ is a Hilbert space then for any two compact subsets $A, B$ of $X$ the following inequality holds

$$
\left\|c_{A}-c_{B}\right\|^{2} \leqslant\left[r_{A}+r_{B}+h(A, B)\right] h(A, B) .
$$

(2) is precise in the sense that it may become an equality for a suitable choice of $A$ and $B$.

REMARK. Theorem 1 remains valid for centers relative to any vector subspace of $X$.

Let $X$ be a uniformly convex Banach space. Define for $0<\delta \leqslant 1$,

$$
\varepsilon(\delta)=\sup \left\{\|x-y\|: x, y \in X,\|x\|,\|y\| \leqslant 1,\left\|\frac{x+y}{2}\right\| \geqslant 1-\delta\right\} .
$$

Then $\varepsilon(\delta)$ is an increasing function and $\varepsilon(\delta) \rightarrow 0$ for $\delta \rightarrow 0$. Also for $a \geqslant \delta$ we have

$$
x, y \in X,\|x\|,\|y\| \leqslant a,\left\|\frac{x+y}{2}\right\| \geqslant a-\delta \text { imply }\|x-y\| \leqslant a \varepsilon\left(\frac{\delta}{a}\right) .
$$

THEOREM 2. If $X$ is a uniformly convex Banach space and if $A$ and $B$ are closed convex subsets of $X$ then

$$
\left\|P_{A} x-P_{B} x\right\| \leqslant\left\|P_{A} x-x\right\| \varepsilon\left(\frac{h(A, B)}{\left\|P_{A} x-x\right\|}\right)+\left\|P_{B} x-x\right\| \varepsilon\left(\frac{h(A, B)}{\left\|P_{B} x-x\right\|}\right)
$$

for every $x \in X$ such that $\left\|P_{A} x-x\right\|,\left\|P_{B} x-x\right\| \geqslant h(A, B)$. Otherwise,

$$
\left\|P_{A} x-P_{B} x\right\| \leqslant 3 h(A, B) \text {. }
$$

If $X$ is a Hilbert space then

$$
\left\|P_{A} x-P_{B} x\right\|^{2} \leqslant\left[\left\|P_{A} x-x\right\|+\left\|P_{B} x-x\right\|\right] h(A, B) \quad \text { for every } x \in X ;
$$

in particular $\left\|P_{A} x-P_{B} x\right\|=O\left(h(A, B)^{1 / 2}\right)$. The last statement is precise in the sense that $O$ cannot be replaced by $o$.

REMARK 1. We do not know if (2) remains valid without the compactness hypothesis. 
REMARK 2. We do not know a sharp estimate of $\left\|c_{A}-c_{B}\right\|$ similar to (2) in the case when $X$ is a uniformly convex Banach space. The estimate one can derive from the proof of Lemma 2.1 in [8] is not sharp in the Hilbert space case.

REMARK 3. We consider the following example to illustrate (5). Let $G$ be a domain in $\mathbf{R}^{n}$ with sufficiently nice boundary $\partial G$ and denote by $H^{1}(G)$ the usual Sobolev space of functions on $G$ with the first derivatives square integrable and by $H^{1 / 2}(\partial G)$ the space of restrictions to $\partial G$ of functions in $H^{1}(G)$. For two nonnegative functions $f, g \in H^{1 / 2}(\partial G)$ denote by $A_{f}, A_{g}$ the subsets of $H^{1}(G)$ of all nonnegative functions with boundary values $f$ and respectively $g$. Then it is easily checked that (with $\left.X=H^{1}(G)\right) h\left(A_{f}, A_{g}\right) \leqslant C\|f-g\|_{H^{1 / 2}(\partial G)}$ and (5) describes the dependence on the boundary data of solutions of the corresponding variational inequality (see [6]). This example is somewhat academic-for concepts of convergence of convex sets more appropriate in the context of variational inequalities, see [16].

3. Proofs. Without loss of generality we can assume that the space $X$ is real.

The proof of Theorem 1 depends on the following lemma.

LEMMA. If $X$ is a Hilbert space and if $A$ is a compact subset of $X$ then for every $v \in X, v \neq 0$, there exists $z \in A$ such that $\left\|z-c_{A}\right\|=r_{A}$ and $\left(v, z-c_{A}\right) \geqslant 0$.

Proof. Otherwise, by compactness of $A$ we could find an $\alpha>0$ such that $\left(v, z-c_{A}\right) \leqslant-\alpha$ for every $z \in A$ with $\left\|z-c_{A}\right\|=r_{A}$. Using compactness again one could also find a $\beta>0$ such that $\left\|z-c_{A}\right\| \leqslant r_{A}-\beta$ for all $z \in A$ such that $\left(v, z-c_{A}\right) \geqslant-\alpha / 2$. For $\lambda>0$ and $z \in A$ we can write

$$
\left\|z-c_{A}+\lambda v\right\|^{2}=\left\|z-c_{A}\right\|^{2}+2 \lambda\left(v, z-c_{A}\right)+\lambda^{2}\|v\|^{2} \leqslant r_{A}^{2}-\lambda^{2}\|v\|^{2}
$$

if $\left(v, z-c_{A}\right) \leqslant-\alpha / 2$ and $\lambda$ is so chosen that $2 \lambda\|v\|^{2} \leqslant \alpha$. In the case when $\left(v, z-c_{A}\right) \geqslant-\alpha / 2$ we get the estimate $\left\|z-c_{A}+\lambda v\right\|^{2} \leqslant\left(r_{A}-\beta / 2\right)^{2}$ provided $\lambda$ does not exceed the positive root of the equation

$$
\|v\|^{2} \lambda^{2}+2\|v\|\left(r_{A}-\beta\right) \lambda+\frac{3}{4} \beta^{2}-\beta r_{A}=0 .
$$

It follows that with a suitable choice of $\lambda>0, A$ is contained in a sphere with center $c_{A}-\lambda v$ and with a radius less than $r_{A}$ which is impossible. Q.E.D.

To prove (2) assume as we may that $r_{A} \geqslant r_{B}$ and that $v=c_{A}-c_{B} \neq 0$. By the lemma there exists $z \in A$ such that $\left\|z-c_{A}\right\|=r_{A}$ and $\left(z-c_{A}, c_{A}-c_{B}\right) \geqslant 0$. Then $h(A, B) \geqslant \operatorname{dist}(z, B) \geqslant\left\|z-c_{B}\right\|-r_{B}$. On the other hand

$$
\begin{aligned}
\left\|z-c_{B}\right\|^{2} & =\left\|z-c_{A}+c_{A}-c_{B}\right\|^{2} \\
& =\left\|z-c_{A}\right\|^{2}+2\left(c_{A}-c_{B}, z-c_{A}\right)+\left\|c_{A}-c_{B}\right\|^{2} \geqslant r_{A}^{2}+\left\|c_{A}-c_{B}\right\|^{2},
\end{aligned}
$$

implying that

$$
\begin{aligned}
\left\|c_{A}-c_{B}\right\|^{2} & \leqslant\left\|z-c_{B}\right\|^{2}-r_{A}^{2}=\left(\left\|z-c_{B}\right\|-r_{A}\right)\left(\left\|z-c_{B}\right\|+r_{A}\right) \\
& \leqslant\left(\left\|z-c_{B}\right\|-r_{B}\right)\left(\left\|z-c_{B}\right\|+r_{A}\right) \\
& \leqslant\left(\left\|z-c_{B}\right\|+r_{A}\right) h(A, B)
\end{aligned}
$$

and (2) follows since $\left\|z-c_{B}\right\|+r_{A} \leqslant r_{A}+r_{B}+h(A, B)$. 
When $A$ and $B$ reduce to single points (2) becomes an equality.

Another example to this effect can be obtained as follows. Let $X=\mathbf{R}^{2}$ and $S$ denote the vertical strip $0 \leqslant x_{1} \leqslant b$. Take $A$ to be the intersection of $S$ with the closed disk about $(0,0)$ with radius $r>0$ and $B$ - the intersection with $S$ of the closed disk with the same radius and the center at $(b, 0)$.

REMARK 4. It can be shown by means of an example that the statement of the lemma is false without the compactness hypothesis.

Proof of THEOREM 2. We begin with few observations which are immediate consequences of the definitions.

(i) $\left|\left\|x-P_{A} x\right\|-\left\|x-P_{B} x\right\|\right| \leqslant h(A, B)$.

This is valid without the assumption of uniform convexity.

(ii) If $A$ and $B$ are convex subsets of $X$ and [C] denotes the closed convex hull of $C$ then $h(A,[A \cup B]) \leqslant h(A, B)$ and $h(B,[A \cup B]) \leqslant h(A, B)$. Again no assumptions on $X$ are needed.

By the triangle inequality we can write

$$
\left\|P_{A} x-P_{B} x\right\| \leqslant\left\|P_{A} x-P_{[A \cup B]} x\right\|+\left\|P_{[A \cup B]} x-P_{B} x\right\|
$$

and by (ii) it suffices to estimate each term on the right-hand side. Hence it is sufficient to consider the case when one of the sets $A$ and $B$ is contained in the other. Suppose that $A \subset B$; then $\left\|P_{A} x-x\right\| \geqslant\left\|P_{B} x-x\right\|$ and by (i) $\left\|P_{A} x-x\right\|-\| P_{B} x$ $-x \| \leqslant h(A, B)$. Since $\left(P_{A} x+P_{B} x\right) / 2$ belongs to $B$ it follows that

$$
\left\|\frac{1}{2}\left(\left(x-P_{A} x\right)+\left(x-P_{B} x\right)\right)\right\| \geqslant\left\|P_{B} x-x\right\| \geqslant\left\|P_{A} x-x\right\|-h(A, B) .
$$

We can now apply (3) with $\delta=h(A, B), a=\left\|P_{A} x-x\right\|$ and with $x, y$ replaced by $x-P_{A} x$ and $x-P_{B} x$, respectively, to obtain the first term on the right-hand side of (4).

If $\left\|P_{A} x-x\right\| \leqslant h(A, B)$ then by (i) $\left\|P_{B} x-x\right\| \leqslant 2 h(A, B)$ and the triangle inequality yields the desired conclusion.

It is of some interest to check (5) directly without an appeal to (4). To this effect we recall that $\left(x-P_{A} x, P_{A} x-y\right) \geqslant 0$ for every $y \in A$, with a similar inequality for $B$. We can write

$$
\begin{aligned}
\left\|P_{A} x-P_{B} x\right\|^{2}= & \left(P_{A} x-P_{A} P_{B} x, P_{A} x-x\right)+\left(P_{A} P_{B} x-P_{B} x, P_{A} x-x\right) \\
& +\left(P_{B} P_{A} x-P_{B} x, x-P_{B} x\right)+\left(P_{A} x-P_{B} P_{A} x, x-P_{B} x\right),
\end{aligned}
$$

which yields (5) by dropping the first and third terms, applying the Schwarz inequality to the second and fourth and observing that $\left\|P_{A} P_{B} x-P_{B} x\right\|, \| P_{B} P_{A} x-$ $P_{A} x \| \leqslant h(A, B)$.

The last assertion of Theorem 2 is checked by taking $X=\mathbf{R}^{2}$ and choosing $x, A, B$ as follows. $x$ is arbitrary, $A=[a, b]$ and $B=\left[a^{\prime}, b^{\prime}\right]$ are two segments of equal length such that $a+b=a^{\prime}+b^{\prime}=2 c$ and the distance between $x$ and $c$ is fixed. Also $a, b, a^{\prime}, b^{\prime}$ are so chosen that the angles $a b x$ and $a^{\prime} b^{\prime} x$ are both $90^{\circ}$. It is easy to verify that $h(A, B)=O\left(\sin ^{2} \alpha\right)$ and that $\left\|P_{A} x-P_{B} x\right\|=O(\sin \alpha)$ where $\alpha$ is the angle between the segments $A$ and $B$. For $\alpha \rightarrow 0$ this gives the desired conclusion. 


\section{REFERENCES}

1. I. Singer, Best approximation in normed linear spaces by elements of linear subspaces, Springer-Verlag, Berlin and New York, 1970.

2. V. L. Klee, The review of a paper by N. A. Rutledge, Math. Rev. 13 (1952), 661.

3. Circumspheres and inner products, Math. Scand. 8 (1960), 363-370.

4. A. L. Harkavi, On the Čebyšev center and the convex hull of a set, Uspehi Mat. Nauk 19 (120) (1964), 139-145.

5. M. S. Brodskii and D. P. Milman, On the center of a convex set, Dokl. Akad. Nauk SSSR 59 (1948), 837-840.

6. J. L. Lions and G. Stampacchia, Variational inequalities, Comm. Pure Appl. Math. 20 (1967), 493-519.

7. J. M. Lambert and P. M. Milman, Restricted Chebyshev centers of bounded subsets in arbitrary Banach spaces, J. Approximation Theory 26 (1970), 71-78.

8. D. Amir and F. Deutsch, Approximation by certain subspaces in the Banach spaces of continuous vector valued functions, J. Approximation Theory 27 (1979), 254-270.

9. $\mathrm{H}$. Krüger, $A$ remark on lower semi-continuity of the set valued metric projection, J. Approximation Theory 28 (1980), 83-86.

10. T. Borwein and L. Kleiner, The Hausdorff metric and Chebyshev centers, J. Approximation Theory 28 (1980), 366-376.

11. F. Deutsch and J. M. Lambert, On continuity of metric projections, J. Approximation Theory 29 (1980), 116-131.

12. J. Mach, Continuity properties of Chebyshev centers, J. Approximation Theory 29 (1980), 223-230.

13. D. Amir and Z. Ziegler, Relative Chebyshev centers in normed linear spaces. I, J. Approximation Theory 29 (1980), 235-252.

14. B. Brosowski, F. Deutsch and G. Nürenberger, Parametric approximation, J. Approximation Theory 29 (1980), 261-277.

15. D. Amir, Chebyshev centers and uniform convexity, Pacific J. Math. 77 (1978), 1-6.

16. E. DeGiorgi, E. Magenes and U. Mosco (editors), Proc. Internat. Meeting on Recent Methods in Nonlinear Analysis (Rome, 1978), Pitagora Editrice, Bologna, 1979, pp. 101-329.

DEPaRTMENT OF Mathematics, UNiversity OF KANSAS, LAWRENCE, KANSAS 66045 